\title{
Numerical modelling of organic waste dispersion from fjord located fish farms
}

\author{
Alfatih Ali • Øyvind Thiem • Jarle Berntsen
}

Received: 28 October 2010 / Accepted: 11 February 2011 / Published online: 21 April 2011

(C) The Author(s) 2011. This article is published with open access at Springerlink.com

\begin{abstract}
In this study, a three-dimensional particle tracking model coupled to a terrain following ocean model is used to investigate the dispersion and the deposition of fish farm particulate matter (uneaten food and fish faeces) on the seabed due to tidal currents. The particle tracking model uses the computed local flow field for advection of the particles and random movement to simulate the turbulent diffusion. Each particle is given a settling velocity which may be drawn from a probability distribution according to settling velocity measurements of faecal and feed pellets. The results show that the maximum concentration of organic waste for fast sinking particles is found under the fish cage and continue monotonically decreasing away from the cage area. The maximum can split into two maximum peaks located at both sides of the centre of the fish cage area in the current direction. This process depends on the sinking time (time needed for a particle to settle at the bottom), the tidal velocity and the fish cage size.
\end{abstract}

Responsible Editor: Phil Peter Dyke

This article is part of the Topical Collection on Joint

Numerical Sea Modelling Group Workshop 2010

\footnotetext{
A. Ali $(\varangle) \cdot \varnothing$. Thiem

Bergen Center for Computational Science, Uni Research,

Thorm $\varnothing$ hlenst gt. 55, 5020 Bergen, Norway

e-mail: alfatih.ali@bccs.uib.no

$\varnothing$. Thiem

e-mail: oyvind.thiem@bccs.uib.no
}

\section{A. Ali $\cdot$ J. Berntsen}

Department of Mathematics, University of Bergen,

Johannes Brunsgate 12, 5008 Bergen, Norway

J. Berntsen

e-mail: jarle.berntsen@math.uib.no
If the sinking time is close to a multiple of the tidal period, the maximum concentration point will be under the fish cage irrespective of the tide strength. This is due to the nature of the tidal current first propagating the particles away and then bringing them back when the tide reverses. Increasing the cage size increases the likelihood for a maximum waste accumulation beneath the fish farm, and larger farms usually means larger biomasses which can make the local pollution even more severe. The model is validated by using an analytical model which uses an exact harmonic representation of the tidal current, and the results show an excellent agreement. This study shows that the coupled ocean and particle model can be used in more realistic applications to help estimating the local environmental impact due to fish farms.

Keywords Fish farms - Aquaculture modelling • Tides $\cdot$ Particle tracking $\cdot$ Waste dispersion • Hydrodynamic modelling

\section{Introduction}

Fish farming is a rapid growing industry, and the trade in seafood is the second largest export industry in Norway after oil and gas. Globally, the fish farming supplies more than $50 \%$ of the fish consumed by human. Due to its high profits, it has become a major attractive market for investors. However, the potential negative impacts due to spreading of diseases and fish lice between the farmed and wild stocks are a major concern. Many wild salmon stocks in Norway have declined over the last 20-30 years. It is questioned if it is at all possible to have both strong wild salmon stocks 
and a growing fish farming industry in the same fjords systems.

The organic waste from fish farming and its effect on the marine ecosystem has lately also made fish farms front-page news. Silvert and Sowles (1996) summarized the major sources of the environmental impact in the vicinity of fish farms. The focus was on dispersion and deposition of the organic matter, mainly fish faeces and uneaten food, where physical and biological disturbances due to the structure of the fish cage were taken into account. A portion of the waste from fish farms may be dissolved and transported as passive concentrations in the water masses. This dissolved waste can in addition to affect the near field be transported over large distances and affect the remote fish farms. There may also be other environmental impacts due to fish farms such as release of toxic chemicals and depletion of dissolved oxygen which may create dead zones (Hargrave et al. 2005). The dispersal of waste water may not necessary be monotonically decreasing from the source but be transported in plumes that are strongly affected by the fish cages blockage of the free flow (Venayagamoorthy et al. 2011).

Modelling waste dispersion and deposition is considered as the most cost-effective tool for estimating the environmental impact of the fish farming industry. However, good aquaculture modelling which provides tools to understand, assess, predict and manage this impact is still immature and needed. In particular, the goal is to develop numerical model tools to answer questions like: Where will the highest concentration of the organic waste be located?, why is it located there? and what is the magnitude of its impact on the local marine ecosystem, farms production rate and benthic habitats? The answers to such questions can help in establishing, maintaining and developing sustainable aquaculture sites.

Hydrodynamic models coupled to particle tracking models are used to study the dispersion of fish farm waste in some works such as Panchang et al. (1997), Dudley et al. (2000) and Doglioli et al. (2004). However, in most of these studies, only depth-averaged two-dimensional versions of the hydrodynamic models are considered. In Doglioli et al. (2004), for instance, the vertically averaged flow is computed by the twodimensional Princeton Ocean Model (Blumberg and Mellor 1987; Mellor 1996) and then used to derive a three-dimensional flow field. Two-dimensional modelling is strong in areas where water masses are homogene, but when the stratification is present, the vertical variation in the horizontal velocity may be significant.

In this work, the three-dimensional hydrodynamical $\sigma$-coordinate model, The Bergen Ocean Model
(BOM), is used to simulate the tidal currents in an idealized fjord. BOM is coupled to a three-dimensional particle tracking routine to estimate the dispersion of the particulate organic matter from a fish cage. The particle tracking routine uses the current field for advection of particles and random movements to simulate the turbulent diffusion; in addition, each particle is given a settling velocity which may be drawn from a probability distribution. The coupled particle-ocean model is validated using a simple analytical model which uses an exact harmonic representation of the tidal current, and the results correspond very well confirming that the model can be used in more complicated studies where factors like the topography variations and stratification will affect the water flow.

The settling velocity of particles varies due their physical and biological characteristic such as size, weight, shape, density, absorption, digestibility and component nature. Fish of different type and size will eat fish feed of different size and composition resulting in faecal pellets of different size and density and are therefore important for the settling velocity of the faecal pellets. Cromey et al. (2002) conducted measurement experiments of the settling velocity for the Atlantic salmon $(3.39 \mathrm{~kg})$ fish faeces. Sediment traps were used under the cage for faeces collection and then immediately transferred to a glass cylinder for testing. They found that the settling velocity was normally distributed with mean $\mu=0.032 \mathrm{~m} \mathrm{~s}^{-1}$ and standard deviation $\sigma=0.011 \mathrm{~m} \mathrm{~s}^{-1}$. For small faecal pellets of size $0.3-2.5 \mathrm{~mm}$ from the sea bream (Sparus aurata L.), Magill et al. (2006) measured mean settling velocity of $0.0048 \mathrm{~m} \mathrm{~s}^{-1}$. When it comes to the settling of food pellets, Chen et al. (1999) found a mean settling velocity of $0.128 \mathrm{~m} \mathrm{~s}^{-1}$. These values are used as typical settling velocities in the experiments.

Jusup et al. (2007) also used particle tracking modelling to estimate the particles dispersion and benthic carbon loading from fish farms. However, in that work, the tidal and residual currents were extracted from a measured current record at the farm site, and therefore, no hydrodynamic model was used to simulate the currents. In their work, it was shown that the maximum concentration region splits into two parts away from the fish cage centre due to the tides as this study confirms can occur. However, this study also argues that this may not always be the case. If for instance the particles reach the bottom fast, the maximum concentration of organic waste is found under the fish cage and decreases monotonically away from the cage area. The dispersion of sinking particles depends on the sinking time, the tidal velocity and the fish cage size. 
The paper is organized as follows: In Section 2, a description of BOM together with the particle tracking routine and its integration with the ocean model is presented. In addition, some numerical results which investigate the particle accumulation at the seabed and its sensitivity to the settling velocity are given. The simple analytical model is described in Section 3 where a pure comparison between the analytical and the coupled models is presented. Further, numerical results investigating the maximum of the particles accumulation and its sensitivity to the settling velocity, tidal velocity, water depth and cage size are described in that section. Results due to interaction between two tidal constituents are investigated in Section 4. A summary of this study is presented in the last section.

\section{The coupled model}

The coupled model consists of BOM which is described in Section 2.1 and the particle tracking routine that uses the Lagrangian approach described in Section 2.2. The full model system is denoted as the coupled model.

\subsection{The Bergen Ocean Model}

$\mathrm{BOM}$ is a terrain-following three-dimensional hydrodynamical numerical ocean model which utilizes the $\sigma$-coordinate in the vertical direction given by the mapping $\sigma=\frac{z-\eta}{H+\eta}$. Here $z$ is the vertical coordinate, $\eta$ is the surface elevation and $H$ is the bottom depth. This version of BOM uses rectangular coordinates in the horizontal grid. However, the model is parallelled so that larger computational domain can be decomposed and run with a very high resolution.

The model uses the mode splitting technique which separates the governing equations into an external mode for simulating the fast moving surface gravity waves and an internal mode to model the slow moving internal gravity waves (see, for example, Berntsen et al. 1981 and Kowalik and Murty 1993). To compute the external mode, the depth integrated equations are used. The numerical method used for advection of momentum and density is a total variation diminishing scheme with a superbee limiter described in Yang and Przekwas (1992).

Nonhydrostatics pressure capabilities have been implemented enabling the model to simulate smallscale physical processes with grid resolution less than $1 \mathrm{~m}$ (Berntsen et al. 2006). The model uses a finite difference method to solve the governing equations, namely the momentum, conservation and continuity equations, with a C-grid discretization technique. The mathematical variables are the velocity field components, density, pressure, temperature and salinity. BOM is implemented in a Fortran 90 code and is freely available at http://www.mi.uib.no/BOM/. For further information of BOM, see the user guide by Berntsen (2004).

A tidal elevation is used as a driving force. The model domain is an idealized fjord of $4 \mathrm{~km}$ length, $1.2 \mathrm{~km}$ width and a constant depth of $100 \mathrm{~m}$ (flat bottom), which are coinciding with the $x$-, $y$ - and $z$-axes, respectively (Fig. 1). The horizontal grid resolution is $\Delta x=\Delta y=100 \mathrm{~m}$ and 21 equi-distant $\sigma$-coordinate layers are used in the vertical. The focus is on the waste discharge from a fish cage with horizontal dimension of $50 \times 50 \mathrm{~m}^{2}$, centred at the grid cell at $(x, y)=$ $(2,000 \mathrm{~m}, 600 \mathrm{~m})$. The western and the eastern boundaries are open, and here seven grid cells wide flow relaxation zones are used. In the flow relaxation zones, the water elevation is updated at each time step according to

$\phi=(1-\alpha) \cdot \phi_{\mathrm{int}}+\alpha \cdot \phi_{\mathrm{ext}}$,

where $\phi_{\text {int }}$ contains the unrelaxed values computed by the model and $\phi_{\text {ext }}$ is specified external value. The relaxation parameter $\alpha$ varies smoothly from 1 at the open boundary to 0 at the innermost cell of the boundary zone. This method is called flow relaxation scheme (FRS) and described in Martinsen and Engedahl (1987). The tidal surface elevation in both FRS zones is equal and given by the form

$\eta(t)=\eta_{0} \cdot \cos (\omega \cdot t-\theta)$.

Here $\eta_{0}$ denotes the tidal elevation amplitude, $\omega=\frac{360}{T}$ is the tidal frequency where $T$ is the tidal period and $\theta$ is the tidal phase. The approximated tidal flow is therefore in the $x$-direction along west-east and has a similar cosine signal with the same frequency and phase as in Eq. 2. No variations in the water density is accounted for, and also the fish cage has no effect on the water flow since we are considering a clean comparison with the analytical model in Section 3. However, in

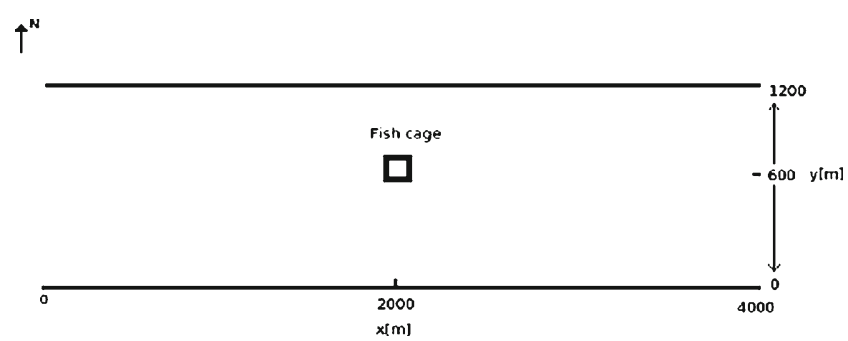

Fig. 1 Horizontal configuration of the model domain (idealized fjord). The west and east boundaries are open 
order to account for the cage effect, a quadratic draglaw could be added to the momentum equations in the $x$ and $y$-directions inside the cage and switched off out side the cage as it is done in Venayagamoorthy et al. (2011). The model is run until all particles have settled on the seabed. The tidal period is $T=12.4206 \mathrm{~h}$ and phase is $\theta=90^{\circ}$.

\subsection{The particle tracking routine}

The particle tracking routine accounts for three processes that influence the particle movement. Advection by the current velocity field, random diffusion due to turbulence and vertical movement towards the sea bed (sinking) caused by the weight of the particles. The particle position $\mathbf{x}(t)=(x(t), y(t), z(t))$ at the $n$-th time step $t_{n}=n \cdot \Delta t$ can then be given by

$$
\begin{aligned}
\mathbf{x}\left(t_{n}\right)= & \mathbf{x}\left(t_{n-1}\right)+\mathbf{U}\left(t_{n-1}\right) \cdot \Delta t+r \cdot \mathbf{U}_{\mathrm{std}}\left(t_{n-1}\right) \\
& \cdot \Delta t-\left(0,0, w_{s}\right) \cdot \Delta t,
\end{aligned}
$$

where $\Delta t$ is the time step, $\mathbf{U}(t)=(U(t), V(t), W(t))$ is the current velocity field in $(x, y, z)$-direction computed by the ocean model, $\mathbf{U}_{\text {std }}(t)$ denotes the standard deviation of $\mathbf{U}(t), w_{s}$ is the particle settling velocity which could be a random variable or takes a single value for all particles and $r$ is a Gaussian distributed stochastic variable with mean $=0$ and standard deviation $=1$.

The particle tracking routine is integrated with the ocean model so that the approximated local flow field from the ocean model is used to propagate the particles at each time step. The velocities at the particles locations are computed using linear interpolation. This is computationally demanding; however, in highresolution studies where the flow field are constantly changing, this can be necessary. Particles that hit the bottom are considered as settled and taken out of the simulation. The particles accumulation at the bottom is computed by counting the particles that hit the bottom within squares of $5 \times 5 \mathrm{~m}^{2}$ areas. Figure 2 shows how the particle model cycle is nested into the ocean model cycle.

The horizontal distance that particles travel before settling on the seabed depends on the water depth, the settling velocity, the local current and the random diffusion. The settling velocities in Chen et al. (1999), Cromey et al. (2002) and Magill et al. (2006) (see Section 1) are used in the numerical experiments in lack of better parameters for mean settling velocity and the standard deviation. In addition, some supplementary settling velocities are utilized for numerical testing and exploring sensitivity.

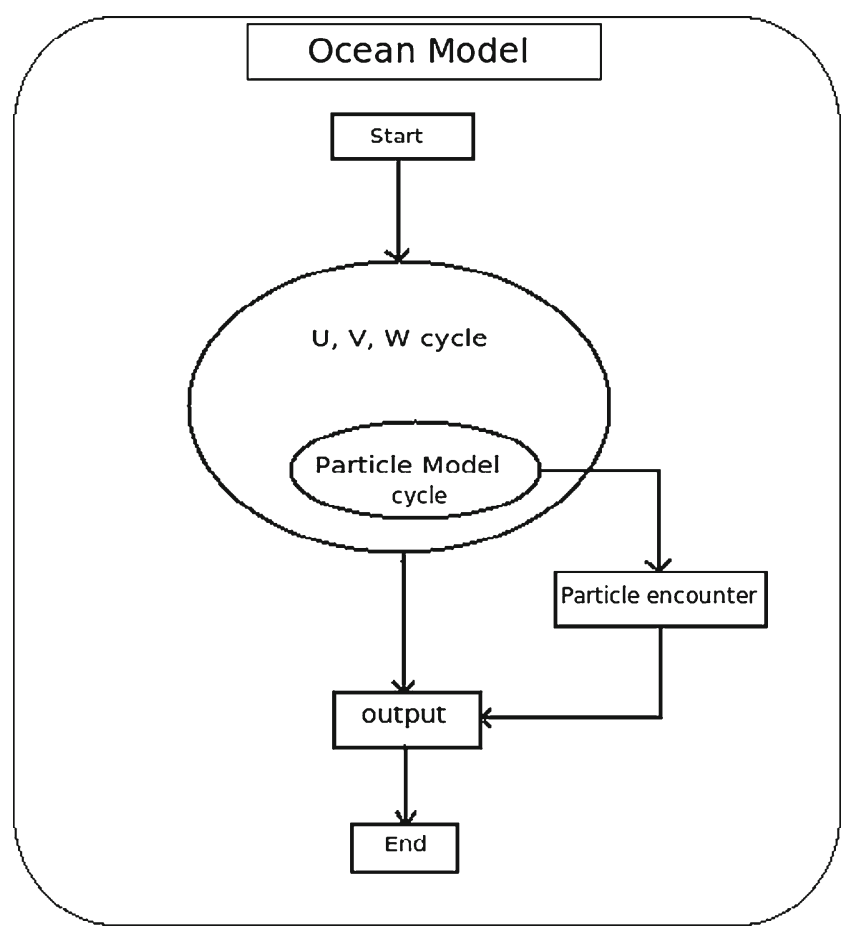

Fig. 2 The flow chart shows the integration of the particle tracking model with the ocean model, and $U, V$ and $W$ represent the velocity components estimated by the ocean model

\subsection{Simulation results}

How the settling velocity affects the particle distributions on the flat bottom (see Fig. 1) is investigated in this section. The initial horizontal position for each particle is chosen randomly with uniform distribution. The particles are released within the cage at $5 \mathrm{~m}$ depth since the fish defecation is observed to occur close to the water surface. The settling velocity $w_{s}$ for each particle is computed randomly using a normal distribution with mean $\mu$ and standard deviation $\sigma$. The tidal elevation amplitude at the open boundaries is $\eta_{0}=0.25 \mathrm{~m}$ which results in a tidal velocity amplitude of $0.025 \mathrm{~m} \mathrm{~s}^{-1}$. The number of particles is $1.62 \times 10^{5}$, and they are released continuously over two tidal periods.

Figure 3 shows the particles footprint at the bottom computed for different means and standard deviations of the settling velocity as variations in the particle characteristics are considered. The results in Fig. 3 could be classified as follows:

1. Particles with mean settling velocity of $0.128 \mathrm{~m} \mathrm{~s}^{-1}$ (mean sinking time is approximately $12 \mathrm{~min}$ ) has one maximum (peak; black line). These particles sink very fast and the influence of the horizontal current is marginal. 
Fig. 3 The number of settled particles (width integrated) for different mean $(\mu)$ and standard deviation $(\sigma)$ of the settling velocity. The experiments are run without random movement. The tidal velocity amplitude is $0.025 \mathrm{~m} \mathrm{~s}^{-1}$, and the cage centre is at $x=2,000 \mathrm{~m}$

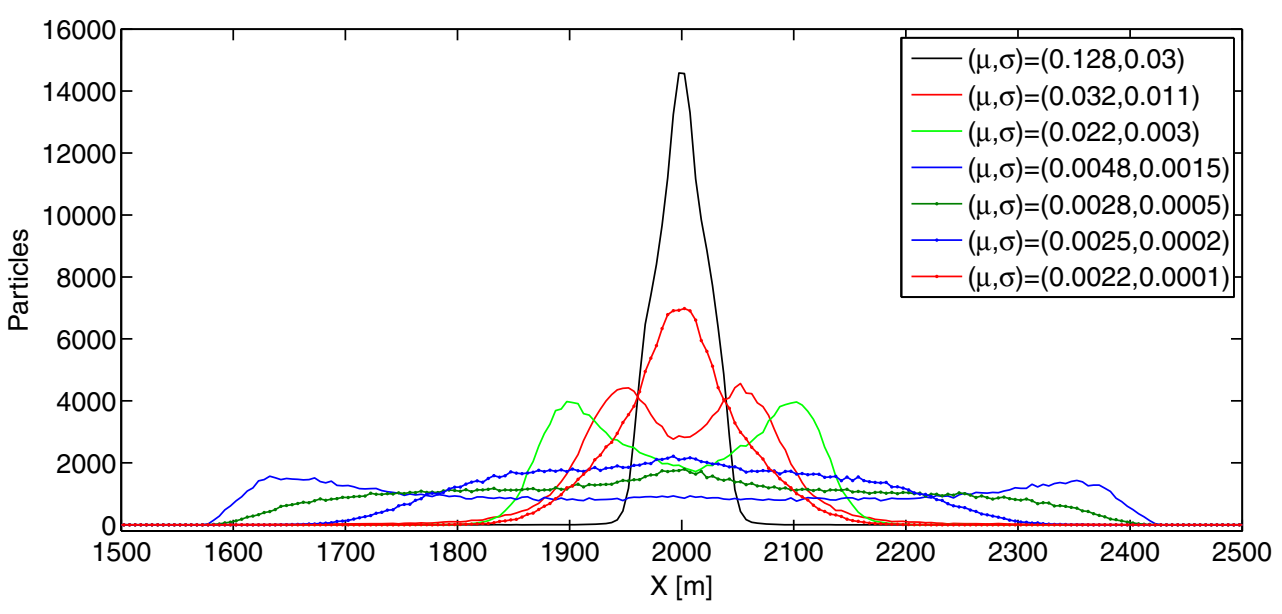

2. For particles with mean settling velocities in the range 0.032 to $0.0048 \mathrm{~m} \mathrm{~s}^{-1}$ (solid lines), the maximum is split into two peaks away from the cage centre. This splitting is due to the mean sinking time ( 0.8 to $5.5 \mathrm{~h})$ approaching $T / 2$, which means that most of the particles will spend longer time in the water column and mainly will be transported in one direction and settle before half of the tidal cycle.

3. For particles with mean settling velocities in the range 0.0028 to $0.0022 \mathrm{~m} \mathrm{~s}^{-1}$ (dot-solid lines), the two peaks merge into one peak beneath the cage centre. This is due to the mean sinking time $(9.4$ to $11.99 \mathrm{~h})$ approaching the full tidal cycle which means that most of the particles will propagate away and then return as the tide reverses. Moreover, they can settle at the same $x$-location as they were released if the sinking time is equal to a multiple of the tidal cycle.

In general, particles that have a sinking time that approaches 0 or close to the tidal period, $T$, attain one maximum located under the fish farm. For particles that have sinking times around $T / 2$, two maximum peaks in the concentration occur, one on each side of the centre of the fish cage, which can result in less accumulation below the fish cage (see Fig. 3).

In the particle tracking routine, the random diffusion is computed from variations in the flow field using the sample standard deviation

$\mathbf{U}_{\text {std }}=\sqrt{\frac{\sum_{i=1}^{N}\left(\mathbf{U}_{i}-\overline{\mathbf{U}}\right)^{2}}{N-1}}$,

where $N$ is the number of the velocity points, $\overline{\mathbf{U}}$ is the mean of the velocity field $\mathbf{U}_{i}$ and $i$ is the index over the velocity points. In the present model setup and forcing, the variations in the velocity are very small due to the simplicity of the model domain (flat bottom and no density variations). This means that the random diffusion has small effects on the particles distribution at the sea bottom (see Fig. 4). There are other ways to implement the random diffusion like using a constant diffusion parameter which would lead to more random variations in the particle distribution, but using Eq. 4 is
Fig. 4 The particle distribution (width integrated): random step (blue circles) vs no random step (red curve), with $\mu=0.032, \sigma=0.011 \mathrm{~m} \mathrm{~s}^{-1}$. The tidal velocity amplitude is $0.025 \mathrm{~m} \mathrm{~s}^{-1}$

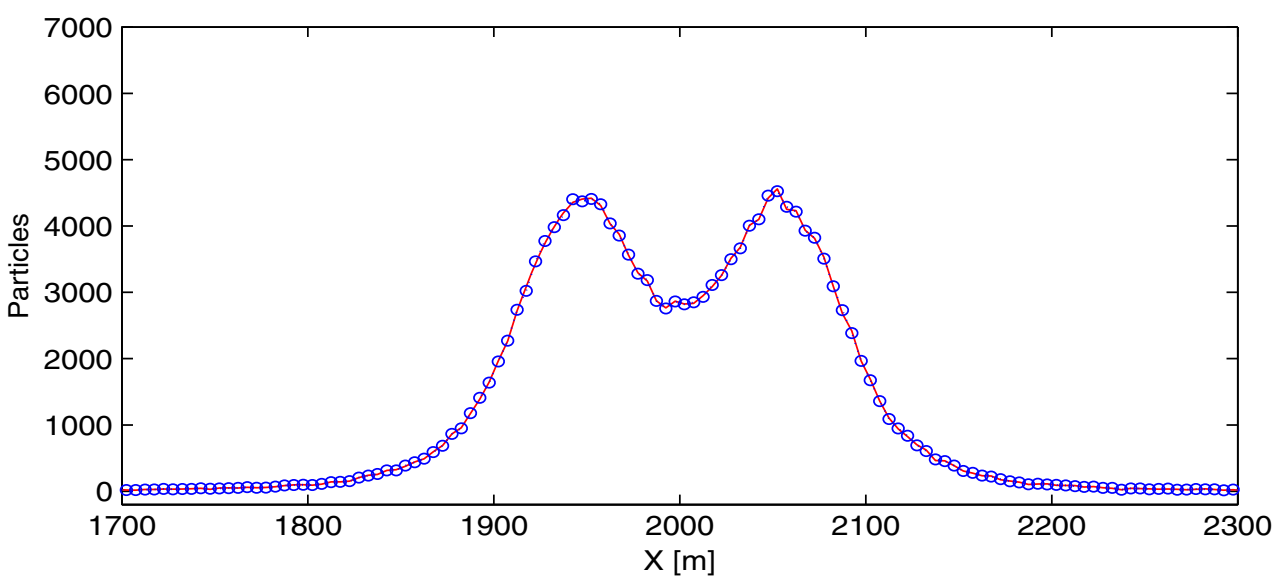


Fig. 5 The particle distribution (width integrated): constant releasing depth of $5 \mathrm{~m}$ (blue line) vs random releasing depth within 0 to $25 \mathrm{~m}$ (red-dash line). The settling velocity is normally distributed with $\mu=0.032, \sigma=0.011 \mathrm{~m} \mathrm{~s}^{-1}$. The tidal velocity amplitude is $0.025 \mathrm{~m} \mathrm{~s}^{-1}$

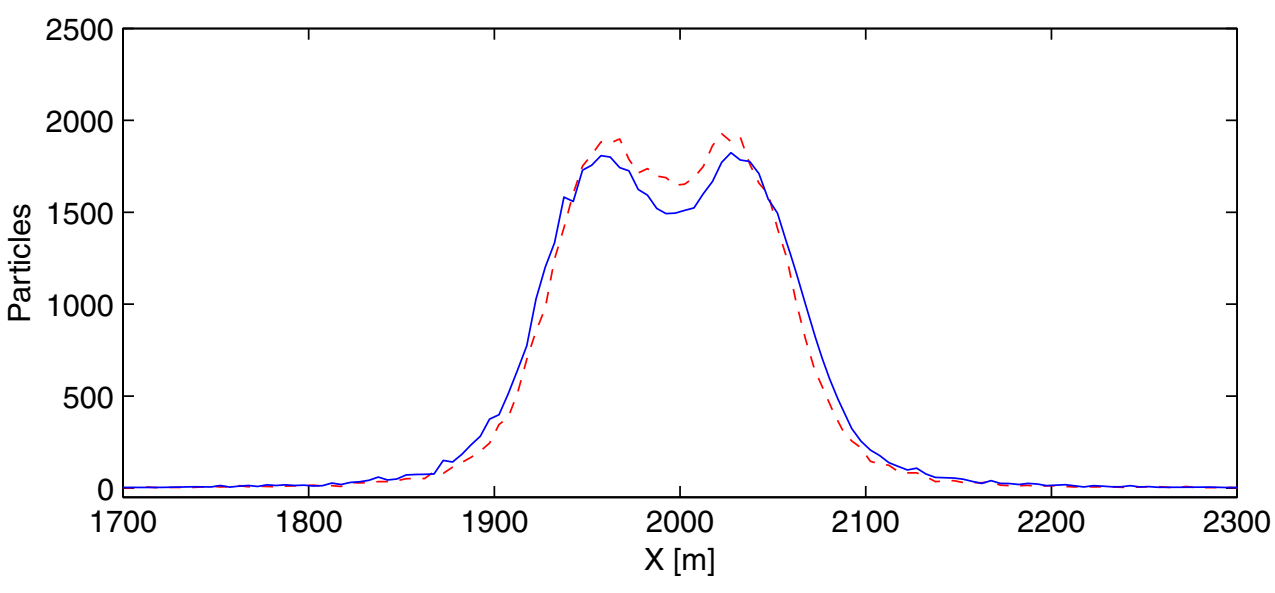

a simple check that the particle tracking routine works satisfactory. The particle tracking experiments to be presented later are run without random diffusion. It can also be noticed from Fig. 4 that the settling velocity is approximately the same as the horizontal velocity, which implied that most particles are transported horizontally by the same order as the water depth.

\subsubsection{Sensitivity to the releasing depth}

Observations have shown that the fish defecation occurs near the water surface (Jusup et al. 2007). Figure 5 shows two numerical experiments, one with a fixed releasing depth of $5 \mathrm{~m}$ and the other with a random releasing depth between 0 and $25 \mathrm{~m}$. The figure demonstrates that the accumulation of the particles under the cage will be reduced when releasing at a constant depth closed to the surface, and the particles footprint beneath the cage is extended when considering random releasing depth because most of the particles will have shorter sinking times as the mean falling depth will be reduced. However, Fig. 5 also shows that using a fixed releasing depth will be sufficient for estimating the footprint extent and maximum and is therefore used in the rest of the simulations.

\section{The analytical model}

The simple model domain and forcing used in the coupled model (Section 2) open for validation through using an exact harmonic representation to simulate the velocity in $x$-direction given by

$u(t)=u_{0} \cdot \cos (\omega \cdot t-\theta)$.

Here $u_{0}$ denotes the tidal velocity amplitude which could be obtained from the tidal velocity in $x$-direction, $U$, from the ocean model. The same tidal frequency and phase as in Section 2 are used. The particle position (without random movements) $(x(t), z(t))$ at the $n$-th time step $t_{n}=n \cdot \Delta t$ can be obtained by

$x\left(t_{n}\right)=x\left(t_{n-1}\right)+u\left(t_{n-1}\right) \cdot \Delta t$,

$z\left(t_{n}\right)=z\left(t_{n-1}\right)-w_{s} \cdot \Delta t$.

For convenience, a single settling velocity, $w_{s}$, is associated for all the particles in the different experiments. If the cage length in the $x$-direction is represented by the interval $[a, b]$, the Eq. 6 can be simplified by directly finding the final horizontal location of any particle at the bottom by integration in time as

$x\left(t_{s}, t_{0}\right)=a+q \cdot(a-b)+\int_{t_{0}}^{t_{0}+t_{s}} u(t) d t$.

Here $t_{0}$ represents the initial time at which a particle is released, $t_{s}$ is the sinking time which is computed as $\frac{H}{w_{s}}$ and $q$ is a random number uniformly distributed between 0 and 1 . The initial horizontal position for each particle is chosen randomly using the parameter $q$. It should be noticed that $H$ is equal to the particles falling depth.

\subsection{Results}

A comparison between the analytical and the coupled model for the particle distribution at the bottom is given in Figs. $6 \mathrm{a}$ and $7 \mathrm{a}$, and it shows an excellent agreement.

Note that when increasing the tidal velocity amplitude from 0.0098 to $0.048 \mathrm{~m} \mathrm{~s}^{-1}$, the single peak in Fig. 6a is split into two peaks in Fig. 7a. The particles settling velocity is $0.032 \mathrm{~m} \mathrm{~s}^{-1}$ (constant for all the particles). The contour lines of the particles distribution on the seabed are shown in Figs. $6 \mathrm{~b}$ and $7 \mathrm{~b}$ using the three-dimensional coupled model, and the color bar shows the number of the settled particles. The location of the maximum of the particles concentration is clearly shown and the number of particles settled under the 
Fig. 6 The particles distribution at the bottom. A total of $1.1178 \times 10^{5}$ particles are continuously released over two tidal cycles with constant settling velocity $w_{s}=0.032 \mathrm{~m} \mathrm{~s}^{-1}$ and tidal velocity amplitude $u_{0}=0.0098 \mathrm{~m} \mathrm{~s}^{-1}$. The cage size is of $50 \times 50 \mathrm{~m}^{2}$ (shown as a black square in $\mathbf{b}$ ), and the vertical depth is $H=95 \mathrm{~m}$

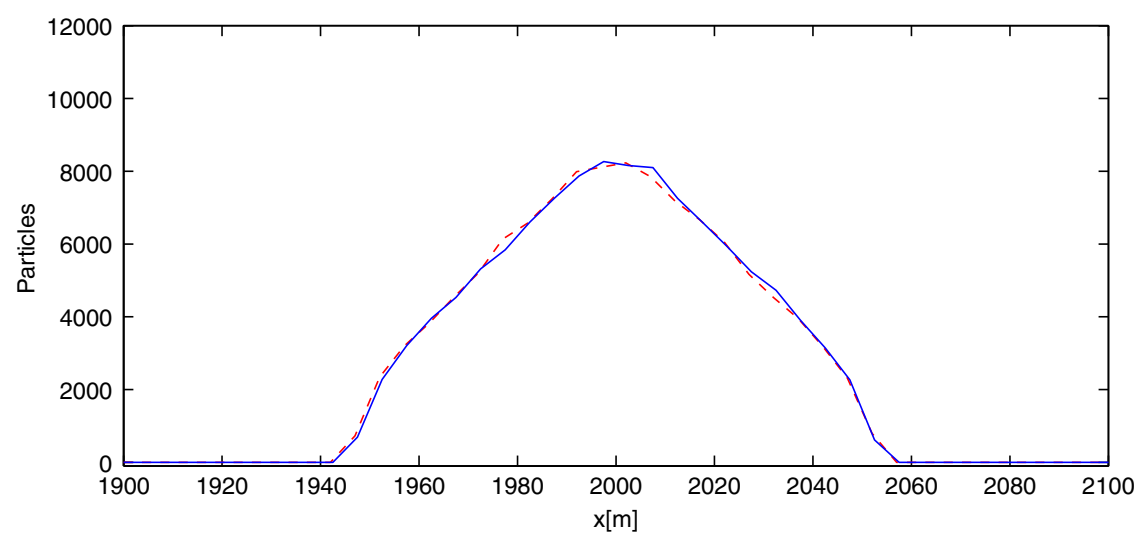

(a) The Coupled Model (width integrated) (blue line) vs the analytical model (red-dash line).

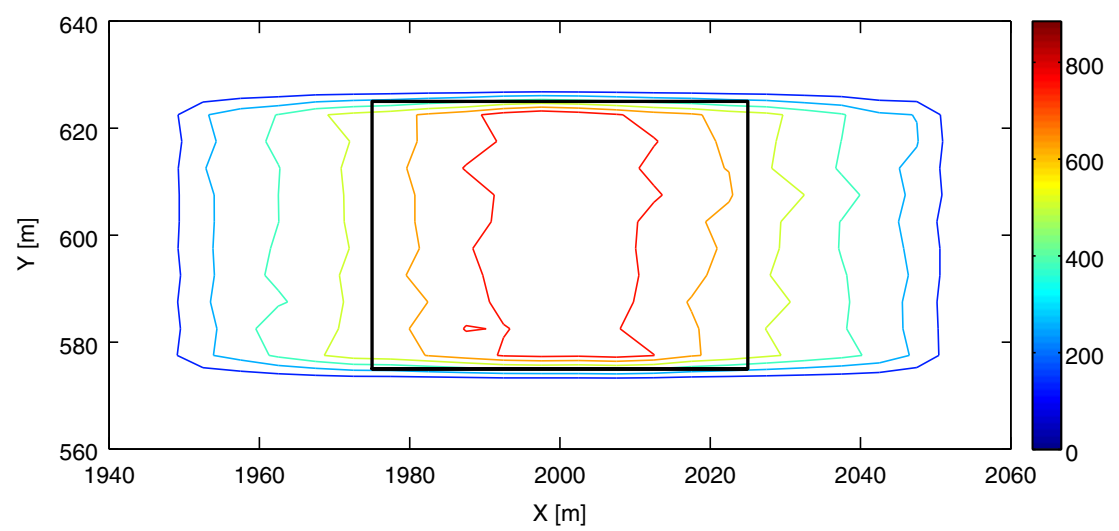

(b) Contour lines of the settled particles in the Coupled Model. The color bar indicates the number of settled particles. centre of the fish farm is decreased by a factor of about one third in Fig. 7. Note also that, since there is no velocity flux in the $y$-direction in the ocean model, the particle distribution is almost zero in the north-south direction.

\subsubsection{Periodic distribution}

The particle distribution is periodic with respect to the sinking time with a period equivalent to the tidal period $T$, which means that the distribution at the seabed for particles with sinking time $t_{s}$ is approximately the same as the distribution for particles with sinking time $t_{s}+T$. This can be seen in Fig. 8 where the particles paths for different sinking times are shown.

For short sinking times $(0.25 \mathrm{~h})$, the particles settle and accumulate just under the fish cage (black lines, Fig. 8 ). As the sinking time increases, the particles are spread away from the cage centre until it approaches a half of the tidal period. This represents the time at which particles can settle at the maximum possible horizontal distance from the cage centre (green lines, Fig. 8). For particles that have longer sinking time than half of the tidal period but less than the full tidal period, the particles will start returning as the tide reverses.

As the sinking time reaches the full tidal period, the particles will settle under the fish cage exactly in horizontal positions at the bottom coinciding with the initial horizontal positions where they were released (blue lines, Fig. 8). This can be seen in Fig. 9 which shows that the footprint for particles with sinking time $t_{s}=0.025 \mathrm{~h}$ is approximately the same as the distribution for particles with sinking time $t_{s}=(0.025+T) \mathrm{h}$.

\subsubsection{One or two maximum concentration regions}

The splitting of the maximum concentration region into two parts was confirmed in a study by Jusup et al. (2007). In their work, however, it was only mentioned that the splitting is due to the tide, but according to this study, that is not the only important factor as we also can find only one maximum under the cage in tidal driven areas.

The particles spatial distribution depends on the settling velocity, the tidal velocity, the water depth and the cage size. A sensitivity analysis of these parameters 
Fig. 7 The particles distribution at the bottom. A total of $1.1178 \times 10^{5}$ particles are continuously released over two tidal cycles with constant settling velocity $w_{s}=0.032 \mathrm{~m} \mathrm{~s}^{-1}$ and tidal velocity amplitude $u_{0}=0.048 \mathrm{~m} \mathrm{~s}^{-1}$. The cage size is of $50 \times 50 \mathrm{~m}^{2}$ (shown as a black square in $\mathbf{b})$, and the vertical depth is $H=95 \mathrm{~m}$

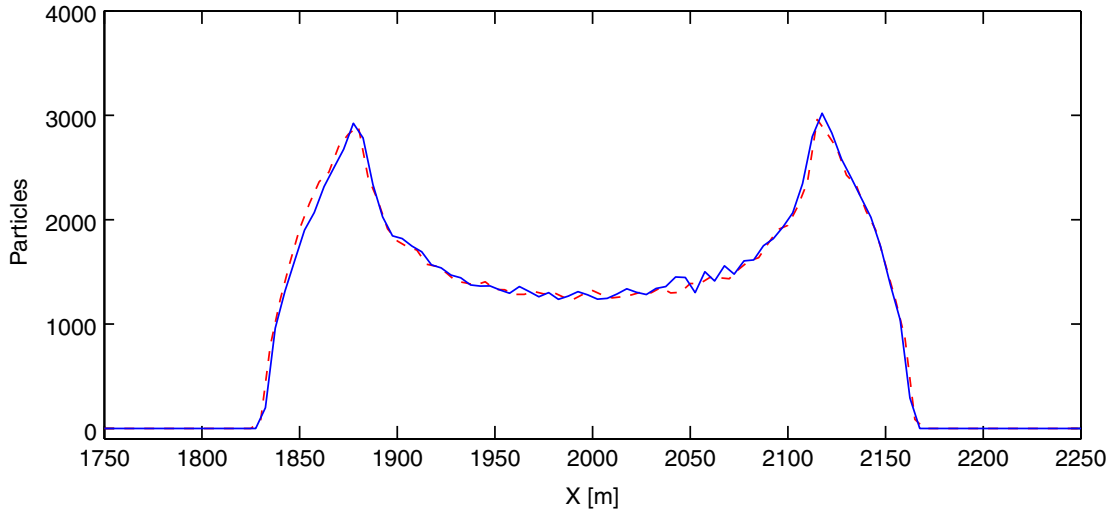

(a) The Coupled Model (width integrated) (blue line) vs the analytical model (red-dash line).

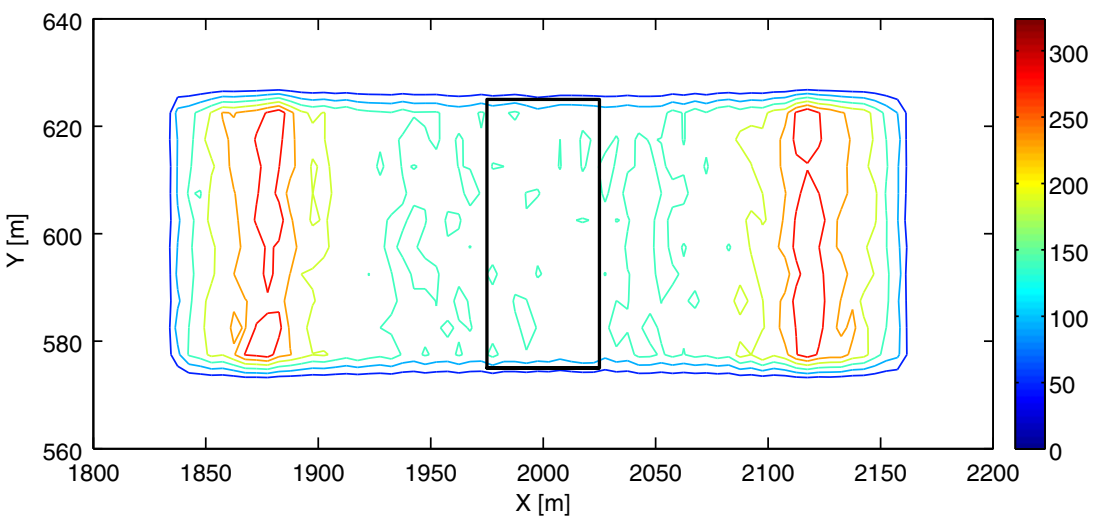

(b) Contour lines of the settled particles in the Coupled Model. The color bar indicates the number of settled particles. is presented in Figs. 10, 11, 12 and 13. Since sinking times $\left(\frac{H}{w_{\mathrm{r}}}\right)$ equal to or longer than the full tidal period will replicate the particles distribution, the focus will be on shorter sinking times than one tidal period. The cage is centred at $x=1,000 \mathrm{~m}$, and $10^{5}$ particles are continuously released over two tidal periods.
Fig. 8 Particles paths for different sinking times $\left(\frac{H}{w_{s}}\right)$ for a tidal velocity amplitude $u_{0}=0.017 \mathrm{~m} \mathrm{~s}^{-1}$. The sinking times are $0.25 \mathrm{~h}$ (black lines), $(0.25+T / 4) \mathrm{h}$ (red lines $)$, $(0.25+T / 2) \mathrm{h}$ (green lines $)$ and $(0.25+T) \mathrm{h}$ (blue lines). The cage size is $b-a=20 \mathrm{~m}$ and the falling depth is $H=100 \mathrm{~m}$

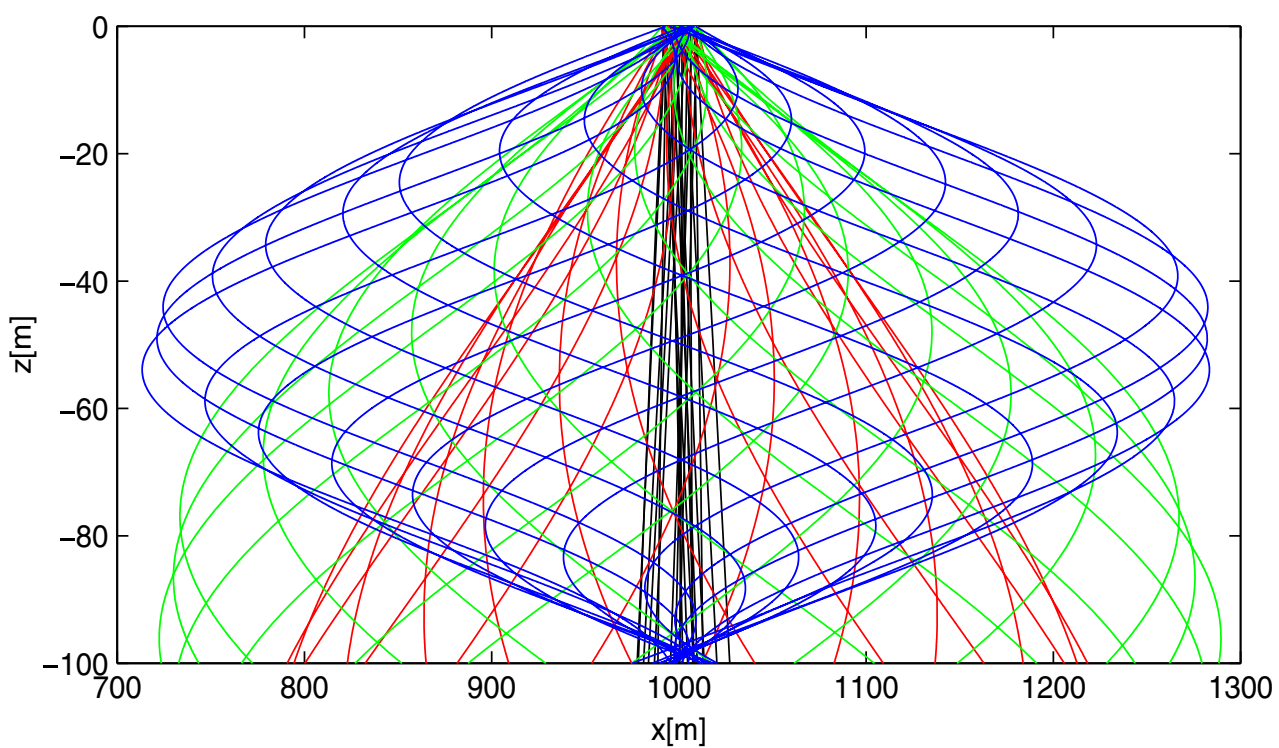


Fig. 9 The footprint of particles with sinking time $t_{s}=0.25 \mathrm{~h}$ (red-circle line $)$ and $t_{s}=(0.25+T) \mathrm{h}$ (blue-solid line). The depth is $H=100 \mathrm{~m}$, the cage size is $b-a=50 \mathrm{~m}$ and is centred at $x=2,000 \mathrm{~m}$ and the tidal velocity is $u_{0}=0.017 \mathrm{~m} \mathrm{~s}^{-1}$

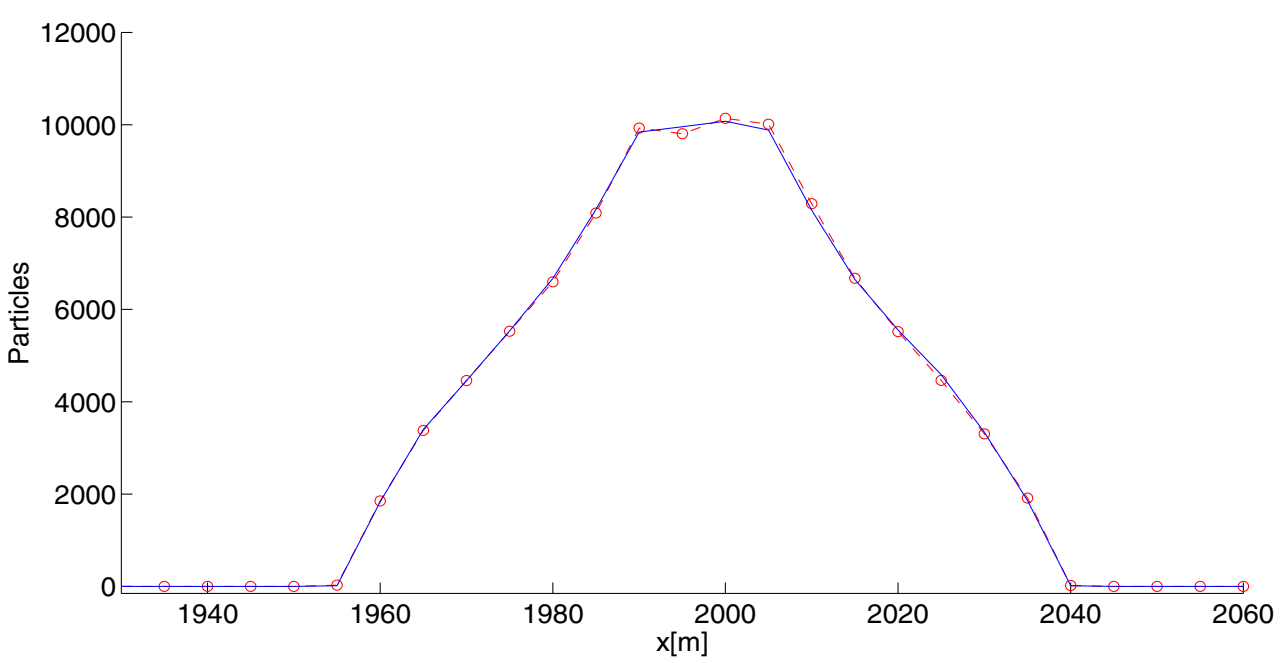

The relation between the cage size $(b-a)$ and the maximum dispersion distance

$d_{\max }\left(H, w_{s}, u_{0}\right)=\max \left\{\int_{t_{0}}^{t_{0}+\frac{H}{w_{s}}} u_{0} \cdot \cos (\omega \cdot t-\theta) d t\right\}$

is the key for the splitting of the maximum peak.

Figure 10 shows that decreasing the settling velocity from $0.032 \mathrm{~m} \mathrm{~s}^{-1}$ to $0.0064 \mathrm{~m} \mathrm{~s}^{-1}$ will increase $d_{\max }$ and when $d_{\max }>\frac{(b-a)}{2}=50 \mathrm{~m}$, the single maximum starts to split into two maximum peaks away from the cage centre.

The same splitting occurs when the tidal velocity amplitude $u_{0}$ is increased from 0.002 to $0.035 \mathrm{~m} \mathrm{~s}^{-1}$ (see Fig. 11). When $d_{\max }>\frac{(b-a)}{2}=50 \mathrm{~m}$, the single peak splits into two.

Deeper water will also lead to splitting since the water depth changes the sinking time. In Fig. 12, the depth is varied between 20 and $100 \mathrm{~m}$ and when $d_{\max }>$ $\frac{b-a}{2}=50 \mathrm{~m}$ the splitting occurs.

The footprint sensitivity to the cage size $(b-a)$ is shown in Fig. 13. For the set of parameters used in this simulation, $d_{\max }=35.98 \mathrm{~m}$, which shows that for cages with sizes less than approximately $72 \mathrm{~m}$, the maximum will start to split into two maximum regions $\left(d_{\max }=\right.$ $35.98 \mathrm{~m}>\frac{b-a}{2}$ ).

The results show that all the studied parameters (tidal velocity, water depth, settling velocity and cage size) are important for dispersing feed and faeces away from a fish farm and that each parameter cannot be looked upon separately when a suited fish farm site is evaluated.

Of special interest, the results show that increasing the cage size to increase the production can result in a maximum concentration of particles beneath the fish cage in an area where earlier, the maximum concentration region was away from the fish cage centre. Accordingly, splitting the larger farms into smaller ones, and
Fig. 10 The particle distribution at the seabed for different settling velocities $w_{s}$. The tidal velocity amplitude is $u_{0}=0.01 \mathrm{~m} \mathrm{~s}^{-1}$, and the falling depth is $H=100 \mathrm{~m}$. The cage size is $b-a=100 \mathrm{~m}$

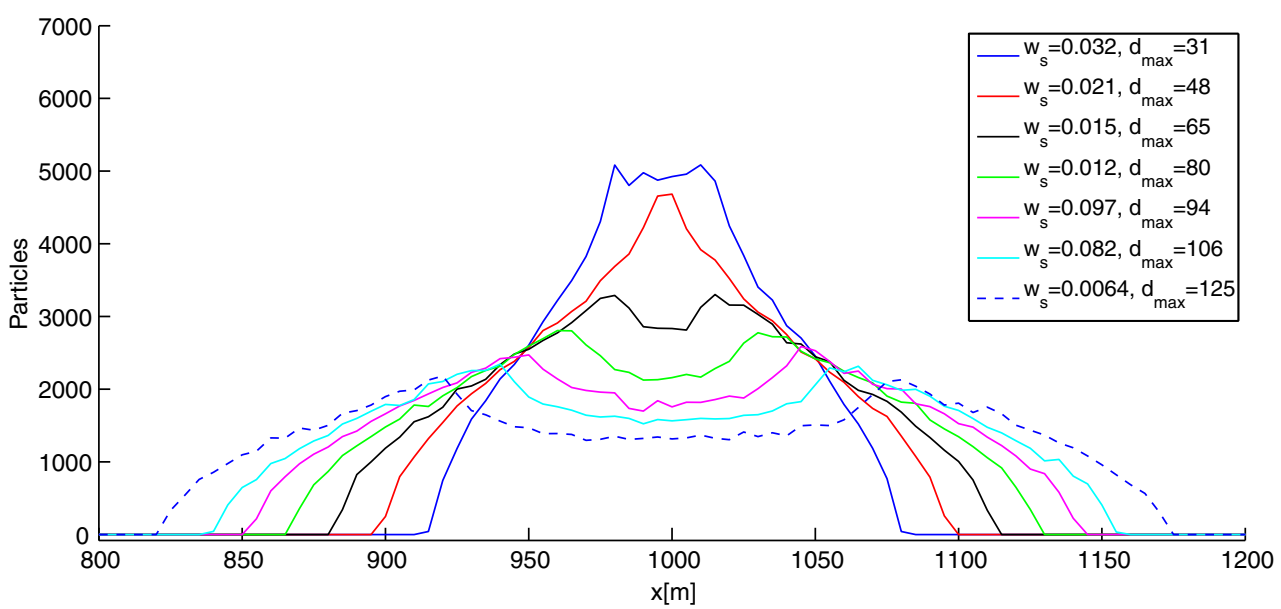


Fig. 11 The particle distribution at the sea bottom for different tidal velocity amplitudes $u_{0}$. The depth is $H=100 \mathrm{~m}$, the cage size is $b-a=100 \mathrm{~m}$, and the settling velocity is $w_{s}=0.032 \mathrm{~m} \mathrm{~s}^{-1}$

Fig. 12 The particle distribution at the sea bottom for different water depths $H$. The tidal velocity amplitude is $u_{0}=0.04 \mathrm{~m} \mathrm{~s}^{-1}$, the settling velocity is $w_{s}=0.032 \mathrm{~m} \mathrm{~s}^{-1}$, and the cage width is $b-a=100 \mathrm{~m}$
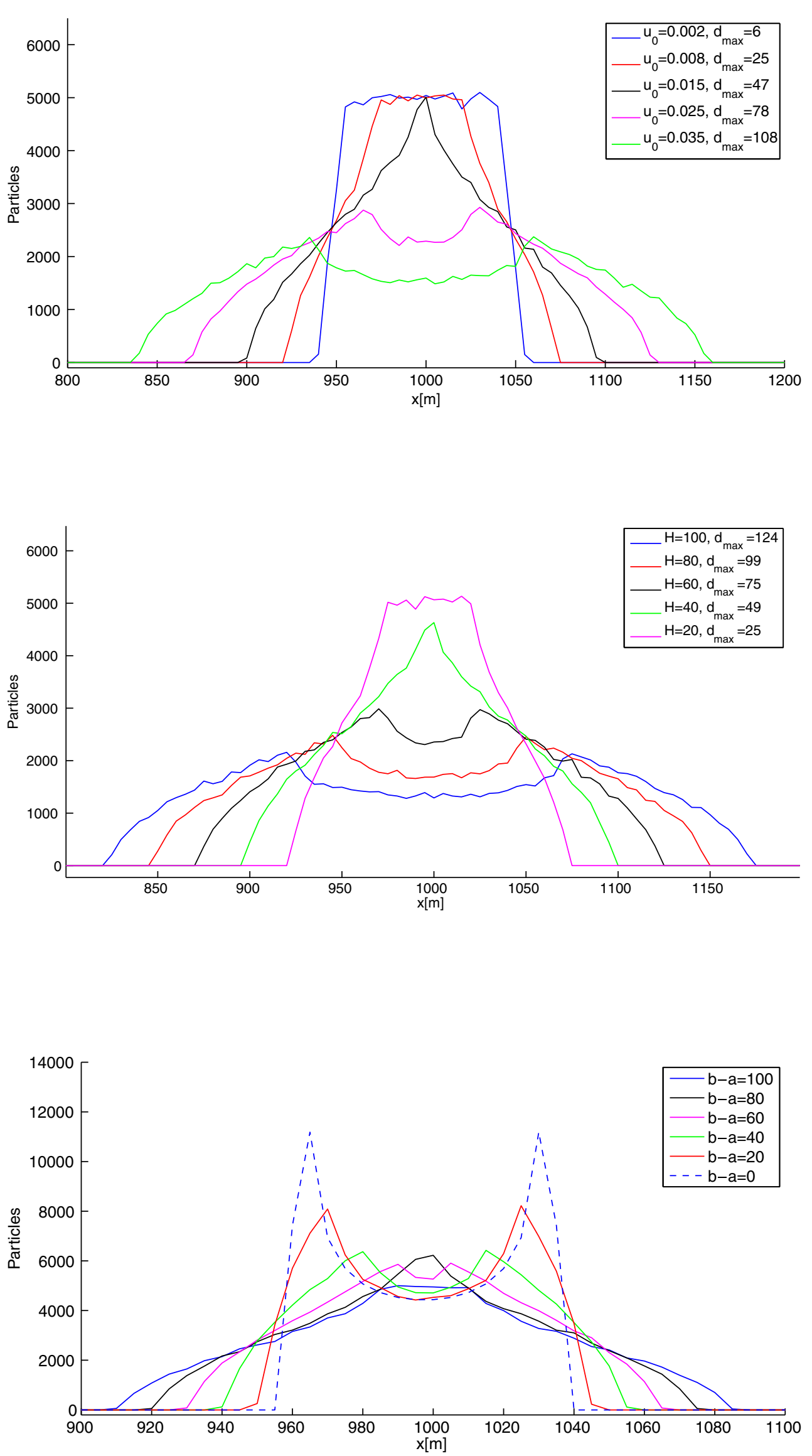

Fig. 13 The particle distribution at the sea bottom for different cage sizes $b-a$. The depth is $H=100 \mathrm{~m}$, the settling velocity is $w_{s}=0.111 \mathrm{~m} \mathrm{~s}^{-1}$, the velocity amplitude is $u_{0}=0.04 \mathrm{~m} \mathrm{~s}^{-1}$, and the maximum dispersion distance is $d_{\max }=35.98 \mathrm{~m}$ 
keeping good distance between them may reduce the environmental impacts since less polluted seabed under the fish farm is expected. However, care must be taken so $d_{\max }$ is larger than half of the cage size and also how fish cages in the proximity interact must be considered since the particles from splitted cages may end up and accumulate under other cages.

To summarize the results in this section: If $d_{\max }\left(H, w_{s}, u_{0}\right)<\frac{(b-a)}{2}$, there will be one maximum concentration point beneath the fish cage centre. If $d_{\max }\left(H, w_{s}, u_{0}\right)>\frac{(b-a)}{2}$, the maximum will split into two maximum concentration peaks away from the fish cage centre. Further, when the difference between the maximum dispersion distance and half of the cage length $\left(d_{\max }\left(H, w_{s}, u_{0}\right)-\frac{(b-a)}{2}\right)$ is quite large, the area under the fish cage may be expected to be less polluted since the dispersion increases. If this difference is small, there will still be two maximum concentration points but closer to the cage centre. In case that the settling velocity is normally distributed, $d_{\max }$ could be computed by setting $w_{s}$ to be equal to the mean settling velocity $\mu$.

\section{Asymmetrical tidal forcing}

The tidal current used to force the model in the previous two sections is symmetrical which has resulted in a symmetrical distribution of the particles on the seabed. However, adding more tidal constituents may produce an asymmetry in the tidal current. If we consider the phase relationship between the M2 and M4 tidal currents shown by Pingree and Griffiths (1979), an asymmetrical current could be obtained as the flood will be stronger while the ebb will be weaker. In this study, this could be done by forcing the model at the open boundaries by a combination of the M2 current

$u_{\mathrm{M} 2}(t)=A_{\mathrm{M} 2} \cdot \cos \left(\omega \cdot t-\theta_{\mathrm{M} 2}\right)$,

and the M4 current

$u_{\mathrm{M} 4}(t)=A_{\mathrm{M} 4} \cdot \cos \left(2 \cdot \omega \cdot t-\theta_{\mathrm{M} 4}\right)$.

Here $\omega$ is the M2 frequency which is equal to $28.984^{\circ} / \mathrm{h}$ (see Stewart 2005), $A_{\mathrm{M} 2}$ and $A_{\mathrm{M} 4}$ are the amplitudes of the M2 and M4 tidal velocities, respectively, while $\theta_{\mathrm{M} 2}$ and $\theta_{\mathrm{M} 4}$ are the corresponding tidal phases. For the asymmetry scenario, we use the phase relationship

$\theta_{\mathrm{M} 4}=2 \cdot \theta_{\mathrm{M} 2}$,

where $\theta_{\mathrm{M} 2}=90^{\circ}$ and $A_{\mathrm{M} 2}=0.025 \mathrm{~m} \mathrm{~s}^{-1}$. More analysis on the phase relationship 11 could be found in Pingree and Griffiths (1979).

Figure 14 shows three numerical experiments of the footprint of the particles on the seabed forced by a combination of the M2 and M4 currents considering the following amplitude relationships:

$A_{\mathrm{M} 4}=0 \cdot A_{\mathrm{M} 2}$,
$A_{\mathrm{M} 4}=0.1 \cdot A_{\mathrm{M} 2}$,
$A_{\mathrm{M} 4}=0.5 \cdot A_{\mathrm{M} 2}$.

The results suggest that there will be a symmetrical footprint when neglecting M4 current (Eq. 12). Using $10 \%$ of the M2 amplitude as an amplitude for M4 (Eq. 13) causes an increase in the concentration of the particles on one side of the cage centre with a decrease in the accumulation on the other. In the last experiment, the use of $50 \%$ of $A_{\mathrm{M} 2}$ as M4 amplitude (Eq. 14) results in the same behaviour as by using Eq. 13 but the asymmetry is increased as a result of the
Fig. 14 The particle distribution (width integrated) using a combination of the M2 and M4 tidal currents considering Eq. 12 in blue line, Eq. 13 in red-dash line and Eq. 14 in black-dash-dot line. The settling velocity is normally distributed with $\mu=0.032$ and $\sigma=0.011 \mathrm{~m} \mathrm{~s}^{-1}$; 55,890 particles are continuously released over two tidal periods. The cage is a square of $50 \times 50 \mathrm{~m}^{2}$ size centred at $(2,000,600) \mathrm{m}$

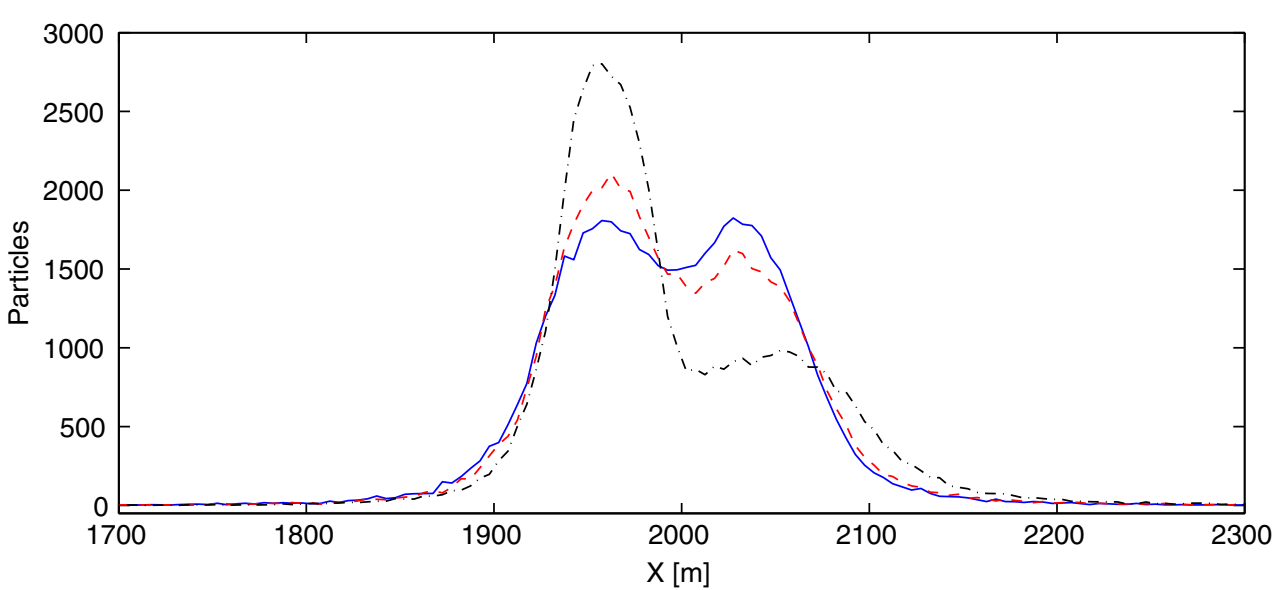


net tidal currents which is quite strong towards west and weaker towards east.

To summarize, the results show that the particles footprint may change when more tidal constituents are included. Several tidal constituents should therefore be included in numerical studies of particles dispersion specially if the dispersion occurs over a relatively short time.

\section{Conclusions}

A three-dimensional particle tracking model is coupled to a three-dimensional $\sigma$-coordinate ocean model and tested in a tidally forced idealized fjord. The particle distributions produced with this coupled model are compared to the particle distribution generated by an analytical model where similar forcing is used, and the result shows a very good agreement.

The results show that when the tide is the only driving force, the maximum concentration area of organic waste depends on the sinking time (water depth and settling velocity), the tidal velocity strength and the fish cage size. Depending on the parameters involved, there may be one maximum accumulation area beneath the cage centre or two maxima, one on each side of the cage centre. A single peak in particle accumulation is found beneath the cage centre for fast sinking particles and for particles where the sinking time is close to a multiple of the tidal period. It is worth noting that for the last type of particles, the maximum concentration point will be located under the fish cage irrespective of the strength of the tide. Particles with sinking times that do not fall under the two categories above will be distributed into two maximum peaks, as is the case in the study by Jusup et al. (2007). Considering the interaction between more tidal components may cause an asymmetrical particle distribution at the seabed.

The study shows also that increasing the cage size increases the likelihood for food and faecal pellets to end up beneath the fish cage which can make the local pollution more severe than the case for smaller fish cages. This might be against the interests of the fish farming industry which is often interested in increasing the production (biomass) by introducing larger fish cages.

Through this study, the performance of the coupled model has been investigated and verified. The model system will now be applied in more realistic studies to improve our understanding of dispersal from fish farms where factors like variations in bottom topography, stratification and forcing (wind, river runoff) are present. Further model studies may be used to help selecting sites for the fish farms to ensure a sustainable and environmentally friendly fish farm production.

The blocking effects of the fish cage and velocities generated by the fishes movement inside the cage can change the free flow in and near the fish cage. These effects can introduce fluid velocities that can influence the sinking velocity of the particulate matter and affect the spreading and plume generation of the dissolved waste. Further, sediment resuspensions in areas where the near bed flow is of significant strength will affect the particle distribution at the seabed. These effects are outside the scope of this work but should be considered in up following studies.

Acknowledgements This research has received support from The Research Council of Norway through NFR 190474/s40 (ECORAIS) and NFR 146526/420 (CORDINO). Thanks to the reviewers for useful comments.

Open Access This article is distributed under the terms of the Creative Commons Attribution Noncommercial License which permits any noncommercial use, distribution, and reproduction in any medium, provided the original author(s) and source are credited.

\section{References}

Berntsen J (2004) Users guide for a modesplit sigma-coordinate numerical ocean model. Tech. rep., Department of Mathematics, University of Bergen

Berntsen H, Kowalik Z, Slid S, Srli K (1981) Efficient numerical simulation of ocean dynamics by splitting procedure. Model Identif Control 2:181-199

Berntsen J, Xing J, Alendal G (2006) Assessment of nonhydrostatic ocean models using laboratory scale problems. Cont Shelf Res 26:1433-1447

Blumberg A, Mellor G (1987) A description of a threedimensional coastal ocean circulation model. Coast Estuar Sci 4:1-16

Chen YS, Beveridge M, Telfer TC (1999) Settling rate characteristics and nutrient content of the faeces of Atlantic salmon (Salmo salar L.) and the implications for modelling of solid wastes dispersion. Aquac Res 30:395-398

Cromey C, Nickell T, Black K (2002) Depomod-modelling the dispersion and biological effects of waste solids from marine cage farms. Aquaculture 214:211-139

Doglioli A, Magaldi M, Vezzulli L, Tucci S (2004) Development of a numerical model to study the dispersion of wastes coming from a marine fish farm in the Ligurian Sea (Western Mediterranean). Aquaculture 231:215-235

Dudley R, Panchan V, Newell C (2000) Application of a comprehensive modeling strategy for the management of net-pen aquaculture waste transport. Aquaculture 187:319-349

Hargrave B, Silvert W, Keizer P (2005) Assessing and managing environmental risks associated with marine finfish farming. Hdb Env Chem 5:433-461

Jusup M, Gecek S, Legovic T (2007) Impact of aquaculture on the marine ecosystem: modelling benthic carbon loading over variable depth. Ecol Model 200:459-466 
Kowalik Z, Murty TS (1993) Numerical modeling of ocean dynamics. Advanced series on ocean engineering, vol 5. World Scientific, Singapore

Magill SH, Thetmeyer H, Cromy CJ (2006) Settling velocity of faecal pellets of gilthead sea bream (Sparus aurata) and sea bass (Dicentrarchus labrax 1.) and sensitivity analysis using measured data in a deposition model. Aquaculture 251:295-305

Martinsen EA, Engedahl H (1987) Implementation and testing of a lateral boundary scheme as an open boundary condition for a barotropic model. Coast Eng 11:603-637

Mellor G (1996) Users guide for a three-dimensional, primitive equation, numerical ocean model. Tech. rep., Princeton University

Panchang V, Cheng G, Newell C (1997) Modeling hydrodynamics and aquaculture waste transport in coastal Maine. Estuaries 20:14-41
Pingree RD, Griffiths DK (1979) Sand transport paths around the British Isles resulting from M2 and M4 tidal interactions. J Mar Bio Assoc UK 59:497-513

Silvert W, Sowles JW (1996) Modelling environmental impacts of marine finfish aquaculture. J Appl Ichthyol 12:7581

Stewart RH (2005) Introduction to physical oceanography. Department of Oceanography, Texas A\&M University, College Station

Venayagamoorthy SK, Ku H, Fringer OB, Chiu A, Naylor RL, Koseff JR (2011) Numerical modeling of aquaculture dissolved waste transport in a coastal embayment. Environ Fluid Mech. doi:10.1007/s10652-011-9209-0

Yang H, Przekwas A (1992) A comparative study of advanced shock-capturing schemes applied to burger's equation. J Comput Phys 102:139-159 